\title{
Plasma Resonance in Layered Normal Metals and Superconductors.
}

\author{
S. V. Pokrovsky" \\ Department of Physics, MIT, Cambridge, MA 02139 \\ and \\ V. L. Pokrovsky \\ Department of Physics, Texas A\& M University, \\ College Station, TX 77843-4242 ? \\ and \\ Landau Institute for Theoretical Physics, \\ Kosygin str. 2, Moscow 117940, Russia.
}

\begin{abstract}
A microscopic theory of the plasma resonance in layered metals is presented. It is shown that electron-impurity scattering can suppress the plasma resonance in the normal state and sharpen it in the superconducting state.
\end{abstract}

\section{Introduction.}

The plasma resonance can be observed as a steep drop of reflectivity when the frequency of an electromagnetic wave goes above the threshold plasma frequency $\Omega$. The latter is determined in an isotropic plasma or a metal by the well-known equation:

$$
\Omega^{2}=\frac{4 \pi n e^{2}}{\mathcal{E} m}
$$

where $n$ is the density of the carriers (electrons), $m$ is their effective mass and $\mathcal{E}$ denotes high frequency dielectric constant due to the inner atomic shells.

If a metal can become superconducting, its London penetration depth $\lambda$ at zero temperature is related to the plasma frequency via a simple relationship:

$$
\Omega=\frac{c}{\lambda \sqrt{\mathcal{E}}}
$$

\footnotetext{
${ }^{1}$ To whom all correspondence should be addressed.

${ }^{2}$ Present address.
} 
where $c$ is the speed of light.

Experimental observation of the plasma resonance in conventional metals is difficult because the plasma frequency usually occurs in the far ultraviolet range, where inner photoeffect processes are substantial. The situation changes drastically for layered metals when the field polarization is perpendicular to the layers. Indeed, one can naively apply eq. (11) with a large effective mass $M$ corresponding to the tunneling motion across the layers. However, we show that the naive approach fails due to the openness of the Fermi-surface. The correct answer is at least $m / M$ times less ( $m$ being a small mass for in-plane motion). Thus the plasma frequency $\Omega_{z}$ for $c$-axis polarization occurs in the far infrared or even in the millimeter range.

Polarized reflection measurements from $\mathrm{YBa}_{2} \mathrm{Cu}_{3} \mathrm{O}_{7}$ crystals have been carried out by Koch et al [1]. For the radiation polarized perpendicularly to the $\mathrm{CuO}_{2}$ planes a striking difference has been discovered between the reflectivity spectra in the normal and in the superconducting state. Typical spectra below the superconducting transition $(90 K)$ show a sudden sharp drop from unity to 0.4 in the vicinity of $12 \mathrm{meV}\left(90 \mathrm{~cm}^{-1}\right)$ at $T=10 \mathrm{~K}$ and to 0.55 in the vicinity of $8 \mathrm{meV}\left(60 \mathrm{~cm}^{-1}\right)$ at $T=65 \mathrm{~K}$ whereas at temperatures above $T_{c}$ reflectivity just decreases smoothly from 0.8 to $0.60-0.65$ in the whole far infrared region $0-15 \mathrm{meV}$.

A similar behavior of the far infrared $c$-polarized reflectivity from the $\mathrm{La}_{2-x} \mathrm{Sr}_{x} \mathrm{CuO}_{4}$ single crystals has been reported by Tamasaku et al [3]. Plasma edge followed by a dip has been observed by them in the superconducting phase at even lower frequencies $\left(25 \mathrm{~cm}^{-1}\right.$ at $x=0.10$ and $50 \mathrm{~cm}^{-1}$ at $x=0.16)$. This spectral feature being very sharp at low temperature becomes smeared when the temperature is increased and eventually disappears in the vicinity of the critical point.

The physical reason for this phenomenon can be readily understood. In the normal state a strong scattering of the electrons by impurities and phonons suppresses the plasma oscillations. However, in the superconducting state there exists a superfluid of Cooper pairs which are not scattered, and thus maintain the coherence of the plasma wave.

The idea of abnormal transparency of layered superconductors has been advocated by T.Mishonov [4]. He pursued a phenomenological approach based on the two-fluid model of superconductivity (Gorter-Kazimir model). 
In this model the dielectric function is postulated in the following form:

$$
\mathcal{E}(\omega)=\mathcal{E}\left(1-\frac{\Omega_{s}^{2}}{\omega\left(\omega+i 0^{+}\right)}-\frac{\Omega_{n}^{2}}{\omega(\omega+i / \tau)}\right)
$$

The first term in the r.h.s. of this equation describes the contribution of the valence electron bands and high frequency optic oscillations. The second term is due to the Cooper pairs and the last one is contributed by the Fermi excitations. The superconducting and the normal plasma frequencies, $\Omega_{s}$ and $\Omega_{n}$, are related to the corresponding BCS densities $n_{s}$ and $n_{n}$ in the same way as in eq. (1). The absorption is characterized by some temperaturedependent attenuation coefficient $1 / \tau$. This approach has been first employed for the analysis of the optical properties of the high- $T_{c}$ superconductors by de Marel et al [2]. A recent theoretical work by Tachiki et al [5] goes along the same lines.

The purpose of the present work is to present a microscopic theory of the plasma resonance in the normal and the superconducting states of layered metals. The anomalously low value of the plasma frequency for the $c$-polarized radiation is a straightforward outcome of this theory. Besides, it provides an interesting and important insight into the role played by elastic scattering of electrons by impurities. The scattering puts down the reflection of electromagnetic waves by destroying the coherence of plasma oscillations in the normal state once the scattering rate is comparable with the plasma frequency. In the superconducting state, however, the frequent scattering prevents the normal carriers to be involved into the collective motion of the superconducting Cooper pairs. Therefore, plasma oscillations of the charged superfluid being virtually independent maintain the reflection below plasma edge.

The paper is organized as follows. We introduce the underlying model in Section 2. The reflectivity in the normal state is evaluated in Section 3 using the kinetic equation. This approach is insufficient to treat the same problem in the superconducting state. Not only the quasi-particle occupation numbers, but also the Cooper pair wave function varies in the electromagnetic wave (see [6], [7]), so that no local dynamics exists for superconductors. An outline of the field-theoretical calculations accounting most naturally for the above-mentioned variations and for the random fields of impurities is drawn in Section 4 . The results of these calculations are represented and discussed in Section 5. We return to the discussion of advantages and limitations of 
our model and summarize its physical implications in the last Section 6 . The analytic continuation of the electromagnetic response function is carried out in Appendix A. The electromagnetic response for the frequencies above the threshold $2 \Delta$ is studied in Appendix B.

Preliminary version of this work has been published in the Proceedings of SPIE conference.

\section{The model.}

Most layered conductors have a rather complicated crystal structure including, in particular, several conducting layers $(\mathrm{CuO})$ per elementary cell. These layers are usually coupled much more strongly than those belonging to different cells. In this article a simplified model with a single layer per elementary cell is employed. To make the model even simpler, the electron dispersion relation is taken to be totally isotropic in the $a b$-plane. Although the spectrum of the real compound possesses only orthorhombic symmetry, the anisotropy in the $a b$-plane is not very pronounced. In other cases the symmetry may be tetragonal. That gives us hope that the isotropic model provides a reasonable approximation.

In our model the electrons are assumed to be scattered predominantly by the impurities. The collisions are assumed to be elastic, and since the overlapping of the electronic wave functions even between the neighboring planes is small, only in-plane scattering is taken into account. In principle, two competing types of processes, that of in-plane and inter-plane scattering with different collision times $\tau$ and $\tau^{\prime}$, respectively, should be considered independently. Note that the inter-plane collision time $\tau^{\prime}$ must be compared to the effective time of inter-plane quantum tunnel hopping suppressed by the in-plane scattering [9]. This time is equal to $\left(\gamma^{2} \tau\right)^{-1}$ where $\gamma$ is an interplane hopping amplitude. We assume the strong inequality $\tau^{\prime} \gg\left(\gamma^{2} \tau\right)^{-1}$ to be valid, allowing us to disregard the inter-plane scattering. Our theory relies on the assumption that the metallic properties of the $\mathrm{CuO}$ planes are not spoiled by scattering, i.e. $\epsilon_{F} \tau \gg 1\left(\epsilon_{F}\right.$ stands for the Fermi energy). On the other hand, the dimensionless parameter $\gamma \tau$ measuring the relative value of the inter-plane tunneling with respect to the in-plane scattering may vary in a broad range. The role of inelastic processes and other more realistic modifications to the theory will be considered elsewhere. 
To summarize, our model is that of non-interacting massive electrons propagating freely in the ab-plane and hopping to the neighboring planes. Their motion in the ideal crystal is governed by the following dispersion law

$$
\epsilon\left(\vec{p}, p_{z}\right)=p^{2} / 2 m+\gamma\left(1-\cos \left(p_{z} d / \hbar\right)\right)
$$

where $\vec{p}=\left(p_{x}, p_{y}\right)$ is an in-plane component of momentum and $p_{z}$ is a quasimomentum component parallel to $c$-axis. The effective mass and the interlayer spacing are denoted by $m$ and $d$ respectively. Finally $\gamma$ is the hopping amplitude introduced earlier.

The following obvious relationship between the hopping amplitude $\gamma$ and the effective mass in $c$-direction $M$

$$
\gamma=\hbar^{2} / M d^{2}
$$

will be employed later.

As far as the superconducting properties are concerned only the simplest ground state characterized by an isotropic and homogeneous distribution of the order parameter is considered. The BCS approximation is adopted. Although the potential drawbacks of the a weak coupling theory for the quantative description of high- $T_{c}$ superconductors are clearly understood, we believe that the basic features of the plasma resonance phenomenon are revealed in the framework of this approach.

\section{Reflectivity in the normal metal.}

We now evaluate the reflectivity for the incident wave propagating along $x$-axis, the surface of the layered metal coinciding with the $y z$-plane, and electric field being normal to the layers, i.e. directed along $z$-axis (c-axis). The current $J_{z}\left(\omega, k_{x}\right)$ induced by the electric field $E_{z}\left(\omega, k_{x}\right)$ in the skin layer is also directed along the $z$-axis:

$$
J_{z}\left(\omega, k_{x}\right)=\sigma_{z z}(\omega) E_{z}\left(\omega, k_{x}\right)
$$

where $\omega$ and $k_{x}$ are the frequency and the wave number of the incident wave, respectively. The conductivity $\sigma_{z z}$ depends in general both on wave number and frequency

$$
\sigma_{z z}\left(\omega, k_{x}\right)=-2 i e^{2} \int \frac{\partial n^{(0)}}{\partial \epsilon} \frac{v_{z}^{2}(\vec{p}) d^{3} p}{\omega+i / \tau-v_{x} k_{x}}
$$


where $n^{(0)}(\epsilon)$ is the Fermi occupation number. Neglecting space dispersion $\left(k v_{F}\right.$ compared to $\left.\omega+i / \tau\right)$ one obtains a kind of Drude-Lorenz formula

$$
\sigma_{z z}(\omega)=\frac{i e^{2} \gamma^{2} m d}{2 \pi \hbar^{4}(\omega+i / \tau)}
$$

Let us note that $\tau$ in the above expression is a true collision time in contrast with the formula for the homogeneous metals where it is substituted by a transport time

Henceforth we will omit tensor and vector indices of the conductivity tensor $\sigma_{z z}$, electric field $E_{z}$, magnetic field $B_{y}$ and wave vector and $k_{x}$ used in the present work and denote them simply $\sigma, E, B$ and $k$, respectively.

The dielectric permeability of the metal is related to the conductivity as

$$
\mathcal{E}(\omega)=\mathcal{E}+\frac{4 \pi i}{\omega} \sigma(\omega)=\mathcal{E}\left(1-\frac{\Omega_{z}^{2}}{\omega(\omega+i / \tau)}\right)
$$

where $\mathcal{E}$ denotes the high-frequency dielectric constant (a contribution due to the valence electrons and high-frequency optical modes), plasma frequency is defined as

$$
\Omega_{z}^{2}=\frac{2 e^{2} m}{\mathcal{E} M^{2} d^{3}}
$$

The dielectric permeability, in addition to being a function of $\omega$, depends on two additional parameters $\beta=v_{F} / c$ and $\Omega_{z} \tau$. Both of them are intrinsic characteristics of the metal. Although $\beta$ is always small, $\Omega_{z} \tau$ may vary in different compounds. We assume that it cannot be too large, obeying the following inequality

$$
\beta \Omega_{z} \tau \ll 1
$$

If (11) is satisfied, the spatial dispersion of the conductivity $\sigma$ may be neglected, as we have done already. Indeed, one can verify that a strong inequality $k v_{F} \ll|\omega+i / \tau|$ is valid where

$$
k=\frac{\omega}{c} \sqrt{\mathcal{E}(\omega)}
$$

provided (11) holds.

\footnotetext{
"The reason for this difference is the cancellation of the "arrival" term in the collision integral due to the independence of the scattering amplitudes on $p_{z}$.
} 
The reflectivity is related to the dielectric constant by the equation

$$
R(\omega)=\left|\frac{\sqrt{\mathcal{E}(\omega)}-1}{\sqrt{\mathcal{E}(\omega)}+1}\right|^{2}
$$

Several typical spectra of the reflectivity are shown in Fig.1. The steep drop of reflectivity appearing in the vicinity of the plasma frequency $\Omega_{z}$ when the latter is large with respect to the scattering rate $1 / \tau$ is usually followed by a dip. The bigger is the high-frequency dielectric constant $\mathcal{E}$, the deeper is the minimum. When the impurity elastic scattering rate $1 / \tau$ is significant, with respect to $\Omega_{z}$, the corresponding reflectivity spectrum becomes featureless.

Let us emphasize two peculiarities of the interaction between the electromagnetic field and layered metals. First, the plasma frequency is surprisingly small. In fact, eq. (10) for $\Omega_{z}^{2}$ may be rewritten as

$$
\Omega_{z}^{2}=\frac{4 \pi N e^{2}}{\mathcal{E} M} \frac{m}{M} \frac{\hbar^{2}}{\left(p_{F} d\right)^{2}}
$$

where $a$ is an in-plane lattice spacing. The above expression for the plasma frequency contains two small factors, $m / M$ and $\left(\hbar / p_{F} d\right)^{2}$, reducing the conventional values by two or three orders. Therefore, it is unlikely that $\Omega_{z} \tau \gg 1$ in experiments on the copper oxides, at least until single crystals of very high quality become available. It looks even less likely that the criterion (11) can be violated. Hence the Drude expression (8) may serve as a good approximation at any frequency. Recall that the additional small factors appear as a consequence of the openness of the Fermi-surface.

The second peculiarity concerns the absence of the so-called anomalous skin-effect for $z$-polarized radiation. In this anomalous regime, a leading role is played by a strong spatial dispersion. The latter might become essential once the mean free path of the electrons exceeds the skin-layer depth. However, in this case the inequality $\beta \Omega \tau \ll 1$ would be violated, whereas it is definitely satisfied in the experiments with high- $T_{c}$ superconductors.

To conclude this section, we argue that all our results are robust with respect to the replacement of the quadratic dispersion $\epsilon(\vec{p})=p^{2} / 2 m$ by some arbitrary anisotropic dispersion $\epsilon(\vec{p})$, provided the Fermi line (in the $a b$ plane) is sufficiently smooth. Indeed, the essential relationship which allows

\footnotetext{
${ }^{4}$ We do not consider $\mathcal{E} \leq 1$.
} 
to neglect spatial dispersion is the inequality $k v_{F} \tau \ll 1$, which is equivalent to the condition (11). The integration along the Fermi-line in (7) gives basically the same formula as for the quadratic dispersion. Only the plasma frequency is redefined:

$$
\Omega_{z}^{2}=2 \pi e^{2} \gamma^{2} d^{2} N\left(\epsilon_{F}\right) /\left(\hbar^{2} \mathcal{E}\right)
$$

where $N(\epsilon)$ is the density of states per unit volume.

\section{Field theory approach.}

For the reason given in the Introduction, we apply field theory methods to the calculation of the dielectric permeability of layered superconductors. If we set the gap in the spectrum, $\Delta$, to zero, we get the result for the normal state. This justification of the kinetic equation by more rigorous field theory methods is useful since the validity of the kinetic equation is not obvious when $\gamma \tau<1$.

The electromagnetic response tensor $Q_{\alpha \beta}$ relates the average current $\vec{J}$ to the vector-potential $\vec{A}$

$$
J_{\alpha}(\omega, \vec{r})=-\int Q_{\alpha \beta}\left(\omega, \vec{r}, \overrightarrow{r^{\prime}}\right) A_{\beta}\left(\omega, \overrightarrow{r^{\prime}}\right) d^{3} r^{\prime}
$$

The quantity $Q_{\alpha \beta}\left(\omega, \vec{r}, \overrightarrow{r^{\prime}}\right)$ is usually called the Pippard kernel. According to the Kubo formula, the response function (Pippard kernel) $Q_{\alpha \beta}\left(\omega, \vec{r}, \overrightarrow{r^{\prime}}\right)$ is equal to the Fourier transform of the retarded Green function of two current densities:

$$
Q_{\alpha \beta}\left(\omega, \vec{r}, \overrightarrow{r^{\prime}}\right)=\frac{i T}{c} \int_{0}^{\infty} d t e^{i \omega t}\left\langle\left[J_{\alpha}(t, \vec{r}), J_{\beta}\left(0, \overrightarrow{r^{\prime}}\right)\right]\right\rangle
$$

where $T$ is the temperature of the system and the angular brackets denote the averaging over the Gibbs ensemble. Following the conventional routine, we first compute the auxiliary Matsubara response

$$
Q_{\alpha \beta}^{M}\left(\omega_{m}, \vec{r}, \overrightarrow{r^{\prime}}\right)=\frac{i T}{c} \int_{0}^{1 / T} d \tau e^{i \omega_{m} \tau}\left\langle J_{\alpha}(\tau, \vec{r}) J_{\beta}\left(0, \overrightarrow{r^{\prime}}\right)\right\rangle
$$

where $\omega_{m}=2 \pi m T$.

The retarded response (16) can be found via analytic continuation of the Matsubara response (17) from the discrete points $\omega=i \omega_{m}$ in the upper 
half-plane of the complex variable $\omega$, using the well-known relation

$$
Q_{\alpha \beta}^{R}\left(i \omega_{m}, \vec{r}, \overrightarrow{r^{\prime}}\right)=Q_{\alpha \beta}^{M}\left(\omega_{m}, \vec{r}, \overrightarrow{r^{\prime}}\right)
$$

If elastic impurity scattering only is taken into account, the Matsubara Green function of currents in (17) may be expressed in terms of two-point electronic Green functions. We prefer to write down the corresponding relation in momentum space rather than in the coordinate space; this will prove convenient for subsequent averaging over impurity configurations:

$$
\begin{array}{r}
Q_{\alpha \beta}^{M}\left(\omega_{m}, \vec{k}, \overrightarrow{k^{\prime}}\right)=-\frac{e^{2}}{c} \int \frac{d^{3} P}{(2 \pi \hbar)^{3}} \frac{\partial^{2} \xi(\vec{P})}{\partial P_{\alpha} \partial P_{\beta}} T \sum_{\nu} G^{M}\left(\eta_{\nu}, \vec{P}_{+}, \vec{P}_{-}\right)- \\
\frac{i e^{2}}{c} \int \frac{d^{3} p}{(2 \pi \hbar)^{3}} \frac{d^{3} q}{(2 \pi \hbar)^{3}} v_{\alpha}(\vec{p}) v_{\beta}(\vec{q}) \Gamma\left(\omega_{m}, \vec{p}_{+}, \vec{p}_{-}, \vec{q}_{+} \vec{q}_{-}\right)
\end{array}
$$

where $\eta_{\nu}=(2 \nu+1) \pi T$, and $\vec{p}_{ \pm}=\vec{p} \pm \vec{k} / 2, \vec{q}_{ \pm}=\vec{q} \pm \overrightarrow{k^{\prime}} / 2, \vec{P}_{ \pm}=\vec{P} \pm\left(\vec{k}-\overrightarrow{k^{\prime}}\right) / 2$, and the group velocity $v_{\alpha}(p)=\partial \xi / \partial p_{\alpha}$. Finally, the vertex $\Gamma$, defined as

$$
\begin{array}{r}
\Gamma\left(\omega_{n}, \vec{p}_{+}, \vec{p}_{-}, \vec{q}_{+}, \vec{q}_{-}\right)=T \sum_{\nu} G^{M}\left(\omega_{n}+\eta_{\nu}, \vec{p}_{+}, \vec{q}_{+}\right) G^{M}\left(\eta_{\nu}, \vec{p}_{-}, \vec{q}_{-}\right)+ \\
T \sum_{\nu} \bar{F}^{M}\left(\omega_{n}+\eta_{\nu}, \vec{p}_{+}, \vec{q}_{+}\right) F^{M}\left(\eta_{\nu}, \vec{p}_{-}, \vec{q}_{-}\right)
\end{array}
$$

depends on the four momenta $\vec{p}_{ \pm}, \vec{q}_{ \pm}$. Let us emphasize that the electronic Green functions are the exact Green functions for the non-interacting electrons moving in the potential of some arbitrary but fixed impurity configuration, and obeying the well-known Dyson integral equation [8]. Averaging over the electronic Fermi distribution has already been performed in (19), but averaging over the random ensemble of impurities is yet to be done using the standard diagrammatic technique [8]. After this second averaging, translational invariance is restored, and the averaged Green functions depend on twice as few variables:

$$
\begin{aligned}
& G^{M}\left(\eta_{\nu}, \vec{p}, \vec{q}\right)=\delta(\vec{p}-\vec{q}) G^{M}(\eta, \xi(\vec{p})) \\
& F^{M}\left(\eta_{\nu}, \vec{p}, \vec{q}\right)=\delta(\vec{p}+\vec{q}) F^{M}(\eta, \xi(\vec{p}))
\end{aligned}
$$

These reduced Green functions were calculated by Klemm et al [10], by a natural modification of the method of averaging originally proposed by Abrikosov and Gor'kov [8] for isotropic superconductors: 


$$
\begin{array}{r}
G^{M}(\eta, \xi)=-\frac{i \eta \tilde{\epsilon}(\eta) / \epsilon(\eta)+\xi}{\tilde{\epsilon}(\eta)^{2}+\xi^{2}} \\
F^{M}(\eta, \xi)=\frac{\Delta \tilde{\epsilon}(\eta) / \epsilon(\eta)}{\tilde{\epsilon}(\eta)^{2}+\xi^{2}}
\end{array}
$$

Here

$$
\epsilon(\eta)=\sqrt{\eta^{2}+\Delta^{2}} \quad \tilde{\epsilon}(\eta)=\epsilon(\eta)+1 /(2 \tau)
$$

The averaging of the vertex (20) normally involves a solution of the BetheSalpeter equation or, more accurately, a system of equations for different vertices. For the dynamic processes the number of components increases since the vertex depends on two independent frequencies and can be retarded or advanced with respect to each of them [11, 8]. Fortunately, these difficulties can be avoided for the component $Q_{z z}$ of the response tensor. The calculations in this case are simplified due to the independence of the amplitudes of the electron scattering by the impurities on the $z$-component of the electronic momentum. In the framework of this approximation for the Bethe-Salpiter diagrams, integration over the $z$-component of the momentum in each of the vertices can be separated and involves the following integrals:

$$
\int_{-\pi / d}^{\pi / d} \frac{d p_{z}}{(2 \pi)} v_{z}\left(p_{z}\right) G^{M}\left(\eta, \xi_{+}\right) G^{M}\left(\eta, \xi_{-}\right)
$$

where $\xi_{ \pm}=\xi \pm(\vec{k}, \vec{v}) / 2$. These vanish 9 because $v_{z}$ is the total derivative of a periodic function of $p_{z}$. According to the above arguments the component of the response tensor $Q_{z z}$ is given by:

$$
Q_{z z}^{M}\left(\omega_{n}, \vec{k}\right)=-\frac{i e^{2}}{c} \int \frac{d^{3} p}{(2 \pi)^{3}} v_{z}^{2}\left(p_{z}\right) \Gamma^{M}\left(\omega_{n}, \xi_{+}, \xi_{-}\right)
$$

where

$$
\begin{aligned}
\Gamma^{M}\left(\omega_{n}, \xi_{+}, \xi_{-}\right)=T \sum_{\nu} & {\left[G^{M}\left(\omega_{n}+\eta_{\nu}, \xi_{+}\right) G^{M}\left(\eta_{\nu}, \xi_{-}\right)\right.} \\
+ & \left.\bar{F}^{M}\left(\omega_{n}+\eta_{\nu}, \xi_{+}\right) F^{M}\left(\eta_{\nu}, \xi_{-}\right)\right]
\end{aligned}
$$

To obtain the Pippard kernel, one must perform the analytic continuation of the quantity $\Gamma$ and then integrate it over $\xi$. The details of this procedure may be found in the Appendix A.

\footnotetext{
"This nullification corresponds to the vanishing of the "arrival" term in the collision integral of kinetic equation.
} 
plasma resonance

\section{Plasma resonance in a superconductor.}

For the specific problem under consideration we neglect spatial dispersion, i.e. the dependence of $Q_{z z}$ on the wave-vector $\vec{k}$. For the dielectric permeability we use eqs. (9) with the following modification:

$$
\mathcal{E}(\omega)=\mathcal{E}-\frac{4 \pi c}{\omega^{2}} Q_{z z}(\omega)
$$

In the superconducting state the penetration depth does not increase to infinity at zero frequency due to the Meissner effect, but it still has its maximum at zero frequency. We have argued in Section 3 that it is much bigger than the mean free path at high frequencies. This condition is satisfied without doubt at low frequencies. The following calculations are similar to the treatment of the high-frequency response of superconductors by Bardeen and Mattis [12 and by Abrikosov, Gor'kov and Khalatnikov [13] . We modified the method of the latter authors to account for the layered structure and the impurity scattering.

After the analytical continuation in the Appendix A (see eq. (A.7)) of the vertex $\Gamma^{M}(\omega)$ in the eq. (24), the integration over $\xi$ is performed explicitly in the eq. (A.13). We express the Pippard kernel $Q_{z z}$

$$
Q_{z z}=\frac{e^{2} \gamma^{2} m d}{4 \pi c \hbar^{4}} K^{R}(\omega, T)
$$

via the dimensionless function $K^{R}(\omega, T)$. The formulae for its real and imaginary part in the frequency range $\omega<2 \Delta$ derived straightforwardly from the eq. (A.13) are presented below:

$$
\begin{gathered}
\mathcal{R} e K^{R}(\omega, T)=\int_{\Delta-\omega}^{\Delta} \frac{\tanh \left(\eta_{+} / 2 T\right) d \eta}{\epsilon_{+}^{2}+(\bar{\varepsilon}+1 / \tau)^{2}}\left[\epsilon_{+}+\frac{\eta \eta_{+}+\Delta^{2}}{\bar{\varepsilon} \epsilon_{+}}\left(\bar{\varepsilon}+\frac{1}{\tau}\right)\right] \\
+\frac{1}{2} \int_{\Delta}^{\infty}\left[\frac{\epsilon+\epsilon_{+}}{\left(\epsilon+\epsilon_{+}\right)^{2}+1 / \tau^{2}}\left(1-\frac{\eta \eta_{+}+\Delta^{2}}{\epsilon \epsilon_{+}}\right)\left(\tanh \frac{\eta_{+}}{2 T}+\tanh \frac{\eta}{2 T}\right)\right. \\
\left.+\frac{\epsilon_{+}-\epsilon}{\left(\epsilon_{+}-\epsilon\right)^{2}+1 / \tau^{2}}\left(1+\frac{\eta \eta_{+}+\Delta^{2}}{\epsilon \epsilon_{+}}\right)\left(\tanh \frac{\eta_{+}}{2 T}-\tanh \frac{\eta}{2 T}\right)\right] d \eta \\
\mathcal{I} m K^{R}(\omega, T)=\frac{1}{2 \tau} \int_{\Delta}^{\infty} d \eta\left[\left(1-\frac{\eta \eta_{+}+\Delta^{2}}{\epsilon \epsilon_{+}}\right)\left(\left(\epsilon_{+}+\epsilon\right)^{2}+\frac{1}{\tau^{2}}\right)^{-1}\right. \\
\left.-\left(1+\frac{\eta \eta_{+}+\Delta^{2}}{\epsilon \epsilon_{+}}\right)\left(\left(\epsilon_{+}-\epsilon\right)^{2}+\frac{1}{\tau^{2}}\right)^{-1}\right]\left(\tanh \frac{\eta_{+}}{2 T}-\tanh \frac{\eta}{2 T}\right)
\end{gathered}
$$


where the notations has been changed as follows

$$
\eta_{+}=\eta+\omega \quad \epsilon=\sqrt{\eta^{2}-\Delta^{2}} \quad \epsilon_{+}=\sqrt{\eta_{+}^{2}-\Delta^{2}} \quad \bar{\varepsilon}=\sqrt{\Delta^{2}-\eta^{2}}
$$

Although the normal limit of the Pippard kernel at finite frequency cannot be extracted from the above relations, we have verified (see discussion on the page 22 following the eq. (B.2)) that

$$
\left.K^{R}(\omega)\right|_{\Delta=0}=\frac{\omega}{(\omega+i / \tau)}
$$

confirming our earlier calculation based on kinetic equation.

Let us analyze the frequency behavior of the kernel in several regimes corresponding to different values of temperature and different levels of scattering rate. The main difference between the superconducting and the normal states is that $Q_{z z}(\omega)$ vanishes for $\omega=0$ in the normal state, but it takes a positive finite value in the superconducting state in conformity with the London relation between the current and the vector-potential. The London penetration depth $\lambda_{z}$ is connected to the value of the kernel $Q_{z z}(0)$ :

$$
\lambda_{z}^{-2}=4 \pi Q_{z z}(0) / c
$$

Two first terms in the eq. (28) for $\mathcal{R} e K^{R}(\omega)$ contribute to the static limit of the Pippard kernel:

$$
K^{R}(0)=\pi \Delta \tau \tanh \frac{\Delta}{2 T}-\Delta^{2} \int_{\Delta}^{\infty} \frac{d \eta \tanh (\eta / 2 T)}{\sqrt{\eta^{2}-\Delta^{2}}\left[\eta^{2}-\Delta^{2}+1 / 4 \tau^{2}\right]}
$$

This London factor vanishes as $\Delta \longrightarrow 0$.

The Pippard kernel may be evaluated exactly in the clean limit :

$$
\lim _{\tau \longrightarrow \infty} K^{R}(\omega, T)=1
$$

Notice that the limiting value doesn't depend on temperature. This result is naturally associated with the suppression of dissipation. The Cherenkov absorption of photons is forbidden since the Fermi velocity is much less than the speed of light. On the other hand breaking of Cooper pairs is kinematically permitted but its rate is proportional to the Bogolyubov coherence factor [14] $\left(u_{\vec{p}+\vec{k}} v_{\vec{p}}-v_{\vec{p}+\vec{k}} u_{\vec{p}}\right)^{2}$ where $\vec{k}$ is the momentum of the photon. For 
the normal skin effect $(\vec{k}=0)$, which we only consider, this factor is equal to zero.

Comparing the clean limit to the static one we come to conclusion that in a weak scattering regime $(\Delta \tau \gg 1)$ and for the temperature relatively close to the transition point the real part of the Pippard kernel grows rapidly from its static value (33) to unity. This onset becomes less pronounced at lower temperatures (Fig 2A). The increase of scattering rate is accompanied by reduction of the mean level of $\mathcal{R} e K(\omega)$ and smothering of its variation in the low frequency range (Fig $2 \mathrm{~B})$.

At higher frequencies there is a plateau $K^{R}(\omega, T) \approx 1$ ending in the vicinity of the threshold $\omega=2 \Delta$. This is the point of a mild logarithmic singularity

$$
\mathcal{R} e K^{R}(\omega, T)=\frac{\pi \tau(\omega-2 \Delta)}{2} \log \left(\frac{2 \Delta-\omega}{\Delta}\right) \tanh \frac{\Delta}{2 T}+\text { regular terms }
$$

where the derivative with respect to $\omega$ becomes logarithmically large. This singularity is closely related to the Cooper pair breaking above the threshold (see discussion on the page 24).

Thus, by changing the temperature one can observe a large variation of the plasma frequency when $2 \Delta$ approaches the bare plasma frequency $\omega_{p}$ but still is larger than the latter. The attenuation can remain reasonably small to provide a sharp plasma resonance since $\Omega, \tau$ and $T_{c}$ are independent parameters.

The observable plasma frequency at a given temperature is defined as a root of equation

$$
\mathcal{R} e \mathcal{E}\left(\omega_{p}\right)=0
$$

In a sufficiently clean superconductor $(\Delta \tau \gg 1)$ and for the moderate values of the bare plasma frequency $\Omega$ (14) the observable plasma frequency $\omega_{p}$ almost coincides with the bare one. However, if $\Omega \tau \leq 1$ the observable plasma frequency $\omega_{p}$ diminishes reaching its static limit at $\omega \ll 1 / \tau$. In more dirty samples the deviation of the observable plasma frequency from the bare one is very significant in the whole range $0 \leq \Omega \leq 2 \Delta$. The diminution of temperature causes the augmentation of the observable plasma frequency. Nevertheless, in a very dirty limit $(\Delta \tau \ll 1)$ the observable plasma frequency is by a factor $\sqrt{\Delta \tau}$ smaller than the bare one even at zero temperature. 
The behavior of the real part of the Pippard kernel is illustrated in Fig.2A $\left(\tau T_{c}=0.1\right)$ and Fig.2B $\left(\tau T_{c}=3.4\right)$. Two plots are presented on both figures: dashed for $T / T_{c}=0.3$ and dashed-dotted $T / T_{c}=0.74$ respectively. Looking at the dirtier case one can see that the observable plasma frequency is less than its static limit $c / \lambda$ at the lower temperature and exceeds this limit at the higher temperature. It would be very instructive to study experimentally the temperature variation of the plasma frequency in more detail and to compare it to the temperature dependence of the London penetration depth in $c$-direction $\lambda_{z}$. The latter may be obtained independently from the measurements of the radio-frequency impedance. Our theory predicts extremely weak temperature dependence of the observable plasma frequency in clean samples whereas this dependence may be fairly remarkable in samples with a moderate concentration of impurities.

The imaginary part of the Pippard kernel is determined by the dissipation. As it has been already mentioned, two types of photon absorption are substantial: the Cherenkov absorption of a photon by a quasiparticle (Landau damping) and the absorption accompanied by the dissociation of a Cooper pair. The former process is forbidden kinematically in the absence of scattering, the latter is suppressed for the photon momentum $\vec{k}=0$. At a finite scattering rate the momentum is not conserved any more, and both processes become possible. The pair dissociation requires the photon frequency $\omega>2 \Delta$. This range of frequencies is considered in Appendix B.

The probability of the Cherenkov absorption is proportional to the Fermi occupation numbers of quasiparticles. These vanish exponentially at $T \longrightarrow 0$. The asymptotic form of $\mathcal{I} m K^{R}$ for $\Delta \gg T$ and $\omega \gg T$ can be readily found from eq.(29):

$$
\mathcal{I} m K^{R}(\omega)=-e^{-\Delta / T} \sinh \left(\frac{\omega}{2 T}\right) K_{0}\left(\frac{\omega}{2 T}\right) \frac{2 \Delta \tau \sqrt{2(2+\omega / \Delta)}}{\left(1+2 \omega \Delta \tau^{2}+\tau^{2} \omega^{2}\right)}
$$

where $\mathrm{K}_{0}$ is a MacDonald function of the zeroth order. Examining it one can find that the dissipation depends substantially on the scattering rate and in a somewhat surprising way. Namely, it becomes especially small provided $\Delta \tau \ll 1$. Even as the temperature approaches to the transition point and the number of the excitations increases the dissipation may continue to be small if the scattering rate is sufficiently high. The physical reason for this phenomenon, as already mentioned in the Introduction, is the decoupling of 
a strongly dampen excitation motion from the collective plasma oscillations of the Cooper pairs. However, the asymptotic formula (37) does not apply in this case.

Several plots of imaginary part of the Pippard kernel versus frequency are shown in Fig.3. A minimum of $\mathcal{I} m K^{R}(\omega)$ occurs at some frequency which depends on $T_{c}, T$ and $\tau$. Several characteristic frequency scales for $\mathcal{I} m K^{R}(\omega)$ can be easily seen in eq. (37). These scales are $T, \Delta, \tau^{-1}$ and $\left(\Delta \tau^{2}\right)^{-1}$. When $T$ is the smallest of the scales the minimum of $\mathcal{I} m K^{R}(\omega)$ is located at $\omega \sim T$ and the minimal value of $\mathcal{I} m K^{R}(\omega)$ may reach the order of unity for the moderate temperatures in the clean case. We also see that $\mathcal{I} m K^{R}(\omega)$ is presumably a linear function of $\omega$ at $\omega \ll T$ and behaves as $\omega^{-1 / 2}$ at $\omega \gg T$, but still $\omega \leq \Delta$. One can readily find numerous types of limiting behavior of $\mathcal{I} m K^{R}(\omega)$ depending on the relationship between above-mentioned scales.

Reflectivity spectra are shown in Fig.4. The plots illustrate the behavior of the spectra with respect to the temperature variation and the dependence on a dimensionless parameter $\tau T_{c}$, intrinsic for a specific sample. The values of two other sample-specific parameters: high frequency dielectric constant and bare plasma frequency are chosen to be $\mathcal{E}=25.0$ and $\Omega_{z} / T_{c}=1.2$ for each of the four plots. Each plot corresponding to some scattering rate $\left(\tau T_{c}=0.1 ; 0.9 ; 1.7 ; 3.4\right)$ is combined from three subplots: a solid curve for the normal metal and two for the superconducting state at temperatures $T / T_{c}=0.3$ and $T / T_{c}=0.74$. At the largest value of the scattering rate (Fig.4A) plasma edges followed by the "beaks" are clearly visible on both subplots below $T_{c}$ while the normal subplot is completely featureless. Subsequent plots show the evolution of the plasma feature for the higher temperature. At the lowest scattering rate $\tau T_{c}=3.4$ (Fig.4D) the normal spectrum is still smeared whereas the superconducting spectra for both temperatures: $T=0.74 T_{c}$ and $T=0.3 T_{c}$ are already close to the limiting collisionless spectrum.

\section{Conclusions.}

We have shown that the plasma resonance can be suppressed in the normal state of a layered metal due to a strong electron-impurity scattering, but it should be restored in the superconducting state since the superconducting

\footnotetext{
${ }^{6}$ We choose the transition temperature as a natural energy scale.
} 
part of the electron liquid is not subject to scattering. Moreover, below $T_{c}$ scattering sharpens the plasma edge because it reduces the attenuation by removing Fermi-excitations from the coherent wave motion.

This feature is common for both the BCS and the two-fluid (GorterKazimir) models. Indeed, the imaginary part of the dielectric function (3) is small for the resonance frequency $\omega_{p}=\Omega_{s}$ provided a strong inequality $\Omega_{n}^{2} \tau \ll \Omega_{s}$ is satisfied. This condition is obviously violated in the vicinity of the superconducting transition.

Let us further discuss the relationship between the present theory and the two-fluid model. Namely, we are going to address the question, to what extent the phenomenological expression (3) may approximate the analytic structure of the real dielectric function which we believe is correctly described by eqs. (26-29). This issue is important since the Kramers-Kronig transformation used routinely in the analysis of the experimental reflectivity data is very sensitive to the singularities of the analytic function involved.

Let us first compare the behavior of the real part of the dielectric function in a weak scattering regime: $\Delta \tau \gg 1$. In this case the BCS behavior described in the previous section is reasonably well reproduced in the frameworks of the two-fluid model. In particular, the static limit of $\omega^{2} \mathcal{R} e \mathcal{E}(\omega)$ is proportional to the density of superconducting Cooper pairs. The growth of the response real part due to the contribution of the normal carriers saturates at the same level as in the BCS model when $\omega \gg 1 / \tau$. However, even in the clean limit the shapes and (which may be more important) the characteristic scales of the low frequency onset are different. Indeed, the intermediate frequency scale $1 / \Delta^{2} \tau$ arising naturally in the BCS theory cannot be found in the Gorter-Kazimir model. Finally, it is impossible to incorporate the threshold phenomena in the vicinity of $\omega=2 \Delta$, like a logarithmic singularity of $\mathcal{R} e K^{R}(\omega, T)$ described by the eq. (35), into the two-fluid plasma electrodynamics.

The augmentation of scattering worsens the agreement between the two models. In a strong coupling regime: $\Delta \tau \ll 1$ the two-fluid model predicts an anomalously long plateau at the level of the static limit because the normal electrons are overdampen and do contribute only to the imaginary part of the Pippard kernel. The BCS strong coupling behavior of the $\mathcal{R} e K^{R}(\omega)$ is different (see Fig. 2B). In fact, the two-fluid model encounters some principal difficulties at small enough $\Delta \tau$. It is not clear how the normal and superfluid densities can be defined consistently when the non-conservation of 
the momentum becomes significant.

In the work [3] the clean limit of the two-fluid model has been considered. This approach looks dubious because the fact that the resonance is suppressed in the normal state combined with the experimental value of $\omega_{p} \sim 2 / 3 \Delta$ imply that $\Delta \tau<1$.

As far as $\mathcal{I} m K^{R}(\omega)$ is concerned one can find less common features than the discrepancies between the two-fluid and the BCS models. It is not surprising, since the imaginary part of the Pippard kernel as a function of $\omega$ has only one characteristic scale, $\tau^{-1}$, whereas it depends on four different scales: $\tau^{-1}, \Delta, T,\left(\Delta \tau^{2}\right)^{-1}$ in the present theory. In particular, the position of the minimum of the function $\mathcal{I} m K^{R}(\omega)$ in the two-fluid theory it is always $\omega_{\text {min }}=\tau^{-1}$. In the BCS calculations it depends strongly on the temperature. At low temperature $\omega_{\min } \approx T$ and is always smaller than $\tau^{-1}$. A striking difference between the BCS and the two-fluid dissipation in a strong scattering regime $\left(\tau T_{c}=0.1\right)$ is demonstrated in Fig. 5A. However, even in the clean superconductor the dissipation calculated in the frameworks of the BCS model is much weaker than its two-fluid counterpart (Fig 5B). Of course the light absorption through the Cooper pair breaking remains beyond the scope of the two-fluid model.

Summarizing, the two-fluid model gives a reasonable, though in some range of variables rather crude approximation for $\mathcal{R} e \mathcal{E}(\omega)$ and fails to describe correctly $\mathcal{I} m \mathcal{E}(\omega)$.

Although many realistic features are lacking in the underlying model we believe that our main findings will survive in its more refined and sophisticated versions. It is likely that the in-plane anisotropy of the electronic dispersion would change the macroscopic constants, but not the shape of the plasma resonance. It is not so clear, however, what would be the impact of the anisotropy of the superconducting gap. One may conjecture e.g. that if the d-wave pairing mechanism of superconductivity prevails the attenuation at low temperatures would not be exponentially small.

In a more ambitious treatment, one may have to reexamine the scattering model. According to the experimental data [15 the resistance in the $c$-direction displays a semi-conducting rather than a metallic behavior. Therefore, the inter-plane motion might be better described in terms of hopping between the defects than between the planes. We argue, however, that at high frequencies long-distance hopping is ineffective, whereas short-range hopping is regulated by inter-plane tunneling. Nevertheless a detailed treat- 
ment of these problems is highly desirable.

Although the plasma resonance has been observed only in the superconducting state so far, future developments in a technology of single crystal preparation could drastically raise the chances of its observation in the normal state. Such single crystals could be utilized as $100 \%$ polarizers in the far infra-red region.

It would be very interesting to search for the plasma resonance in the more pronounced layered superconductors, such as Tl- and Bi-based oxides, where the edge could occur in the microwave region. Especially promising would be epitaxially grown multilayers, in which the transition temperature and the energy gap are determined by the superconducting layers whereas the plasma frequency is determined by the square of the inter-plane tunneling amplitude.

Acknowledgments. We are indebted to P.A. Lee for attracting our attention to the work [3] and to T. Mishonov and L.N. Bulayevskii for useful discussions. Our special thank is due to I. Bozovic and W. Saslow for their interest in this work and attentive reading of the manuscript.

\section{Appendix A Analytical continuation.}

According to Section 1 (eq. (18)), to calculate the Pippard kernel one must perform an analytic continuation of the quantity $\Gamma$ defined by eq. (20) which is reproduced here for visibility omitting the factor $-2 i e^{2} / c$ :

$$
\begin{aligned}
\Gamma^{M}\left(\omega_{n}, \xi_{+}, \xi_{-}\right) & =T \sum_{\nu} G^{M}\left(\omega_{n}+\eta_{\nu}, \xi_{+}\right) G^{M}\left(\eta_{\nu}, \xi_{-}\right) \\
& +T \sum_{\nu} \bar{F}^{M}\left(\omega_{n}+\eta_{\nu}, \xi_{+}\right) F^{M}\left(\eta_{\nu}, \xi_{-}\right)
\end{aligned}
$$

from a discrete set of points $\omega_{n}=i 2 n \pi T$ with $n \geq 0$ into the whole upper half-plane of the complex variable $\omega$. We have restored the spatial dispersion omitted in the text, since the analytic continuation can be performed in a more general context.

Once analytic continuation is performed the integration over $\xi$ is in turn. Let us note, however, that the integral over $\xi$ taken in the infinite limits and the sum over $\nu$ together are divergent. Instead of introducing an explicit cutoff in course of calculations one can deal with convergent expressions 
plasma resonance

employing a proper regularization procedure. To this end we subtract from $\Gamma(\omega)$ the same quantity at $\omega=0$ and $\Delta=0$. The corresponding Green functions will be labeled by an additional subscript 0 .

We start from the analytical continuation of the Green functions calculated by Klemm et al (22,23) from a discrete set of arguments $\eta_{\nu}=i \pi(2 \nu+1)$. First, we rewrite these functions in the following form:

$$
\begin{array}{r}
G^{M}(\eta, \xi)=-\frac{i(\eta+\eta /(\epsilon(\eta) \tau))+\xi}{(\epsilon(\eta)+1 / 2 \tau)^{2}+\xi^{2}} \\
F^{M}(\eta, \xi)=\frac{\Delta\left(1+(\epsilon(\eta) \tau)^{-1}\right)}{(\epsilon(\eta)+1 / 2 \tau)^{2}+\xi^{2}}
\end{array}
$$

Expanding the above expressions over the poles of the formal variable $\epsilon(\eta)$ and analytically continuing the latter as a function of the variable $\eta$ one obtains the retarded Green functions in the following form:

$$
\begin{array}{r}
G^{R}(\eta, \xi)=\frac{1}{2}\left(\frac{1+\eta / \epsilon_{R}(\eta)}{\epsilon_{R}(\eta)+i / 2 \tau-\xi}-\frac{1-\eta / \epsilon_{R}(\eta)}{\epsilon_{R}(\eta)+i / 2 \tau+\xi}\right) \\
F^{R}(\eta, \xi)=\frac{1}{2} \frac{\Delta}{\epsilon_{R}(\eta)}\left(\frac{1}{\epsilon_{R}(\eta)+i / 2 \tau-\xi}+\frac{1}{\epsilon_{R}(\eta)+i / 2 \tau+\xi}\right)
\end{array}
$$

where

$$
\epsilon_{R}(\eta)=\sqrt{(\eta+i 0)^{2}-\Delta^{2}}
$$

The branch of the square root is chosen to give positive values of $\epsilon_{R}(\eta)$ for $\eta>\Delta$. The infinitesimal imaginary number is added to its argument in order to prevent the branching points and the poles of the above functions from entering the upper half plain of the complex variable $\eta$. The advanced Green functions can be obtained from the retarded one by a formal substitution $\tau \rightarrow-\tau$ and $\epsilon_{R}(\eta) \rightarrow \epsilon_{A}(\eta)$ where

$$
\epsilon_{A}(\eta)=\sqrt{(\eta-i 0)^{2}-\Delta^{2}}
$$

According to the above consideration in Section 4 the component of the response tensor $Q_{z z}$ is given by the following expression

$$
Q^{M}\left(\omega_{n}, \vec{k}\right)=-\frac{i e^{2}}{c} \int \frac{d^{3} p}{(2 \pi)^{3}} v_{z}^{2}\left(p_{z}\right) \Gamma\left(\omega_{n}, \xi_{+}, \xi_{-}\right)
$$


where $v_{z}=(2 \pi \gamma / d) \sin \left(2 \pi \gamma p_{z} / d\right)$.

Keeping the external frequency in the subset of discrete imaginary numbers: $\omega=i \omega_{n}=i 2 \pi n T$ we can transform the sum in the r.h.s. of eq. A.1) into an integral:

$$
\begin{aligned}
K^{R}\left(i \omega_{n}\right)= & \frac{1}{4 \pi i} \int_{\Gamma_{1}} G^{R}\left(\eta+i \omega_{n}, \xi_{+}\right) G^{R}\left(\eta, \xi_{-}\right) \tanh \left(\frac{\eta}{2 T}\right) d \eta+ \\
& \frac{1}{4 \pi i} \int_{\Gamma_{2}} G^{R}\left(\eta+i \omega_{n}, \xi_{+}\right) G^{A}\left(\eta, \xi_{-}\right) \tanh \left(\frac{\eta}{2 T}\right) d \eta+ \\
& \frac{1}{4 \pi i} \int_{\Gamma_{3}} G^{A}\left(\eta+i \omega_{n}, \xi_{+}\right) G^{A}\left(\eta, \xi_{-}\right) \tanh \left(\frac{\eta}{2 T}\right) d \eta
\end{aligned}
$$

where the contours $\Gamma_{1}, \Gamma_{2} ; \Gamma_{3}$ in the complex plane $\eta$ go around the intervals of the imaginary axis from $\eta=i 0$ to $\eta=i \infty$, from $\eta=-i \omega_{n}$ to $\eta=i 0$ and from $\eta=-i \infty$ to $\eta=-i \omega_{n}$ respectively, all counterclockwise. These contour integrals can be readily transformed into the integrals along the real axis, using the periodicity of $\tanh (\eta / 2 T)$ with the period $\omega_{n}$. The last step is to put $\omega+i 0$ instead of $i \omega_{n}$ in all integrals to get the "retarded" analogue of the vertex (A.1) written in the following integral form

$$
\begin{gathered}
\Gamma^{R}\left(\omega, \xi_{+}, \xi_{-}\right)=\int_{-\infty}^{\infty} \frac{d \eta}{4 \pi i}\left[\tanh \left(\frac{\eta_{+}}{2 T}\right)-\tanh \left(\frac{\eta}{2 T}\right)\right] \tilde{\Gamma}^{R A}\left(\omega, \eta, \xi_{+}, \xi_{-}\right) \\
\int_{-\infty}^{\infty} \frac{d \eta}{4 \pi i} \tanh \left(\frac{\eta}{2 T}\right)\left[\tilde{\Gamma}^{R R}\left(\omega, \eta, \xi_{+}, \xi_{-}\right)-\tilde{\Gamma}^{A A}\left(\omega, \eta, \xi_{+}, \xi_{-}\right)\right]
\end{gathered}
$$

where double-retarded, double-advanced and mixed vertices are defined by the following equations:

$$
\begin{aligned}
& \tilde{\Gamma}^{R R}\left(\omega, \eta, \xi_{+}, \xi_{-}\right)= G^{R}\left(\eta_{+}, \xi_{+}\right) G^{R}\left(\eta, \xi_{-}\right)- \\
& G_{0}^{R}\left(\eta, \xi_{+}\right) G_{0}^{R}\left(\eta, \xi_{-}\right)+\bar{F}^{R}\left(\eta_{+}, \xi_{+}\right) F^{R}\left(\eta, \xi_{-}\right) \\
& \tilde{\Gamma}^{A A}\left(\omega, \eta, \xi_{+}, \xi_{-}\right)= G^{A}\left(\eta, \xi_{+}\right) G^{A}\left(\eta_{-}, \xi_{-}\right)- \\
& G_{0}^{A}\left(\eta, \xi_{+}\right) G_{0}^{A}\left(\eta, \xi_{-}\right)+\bar{F}^{A}\left(\eta, \xi_{+}\right) F^{A}\left(\eta_{-}, \xi_{-}\right) \\
& \tilde{\Gamma}^{R A}\left(\omega, \eta, \xi_{+}, \xi_{-}\right)= G^{R}\left(\eta_{+}, \xi_{+}\right) G^{A}\left(\eta, \xi_{-}\right)+\bar{F}^{R}\left(\eta_{+}, \xi_{+}\right) F^{A}\left(\eta, \xi_{-}\right)
\end{aligned}
$$

Here and in what it follows $\eta_{ \pm}=\eta \pm \omega$. The expressions (A.9 A.11 are obviously analytic in the upper half-plane and since all the integrals are now convergent, one can safely perform the integration of it over $\xi$ first. This integration becomes especially easy provided the representation (A.3) and 
plasma resonance

its analogue for advanced Green functions are used. The contribution due to the subtracted terms vanishes since

$$
\begin{aligned}
G_{0}^{R}(\eta, \xi) & =\frac{1}{\eta+i / 2 \tau-\xi} \\
G_{0}^{A}(\eta, \xi) & =\frac{1}{\eta-i / 2 \tau-\xi}
\end{aligned}
$$

The result of integration of vertex $\Gamma^{R}\left(\omega, \xi_{+}, \xi_{-}\right)$(see eq. (A.8)) over $\xi$ depends on the scalar product $\vec{k} \vec{v}$. The remaining integration over $\phi$ and $p_{z}$ becomes trivial, once the spatial dispersion is neglected, leading to the following final expression:

$$
\begin{aligned}
K^{R}(\omega) & =\int_{-\infty}^{\infty} \frac{d \eta}{4}\left\{\left(1+\frac{\eta_{+} \eta+\Delta^{2}}{\epsilon_{R}\left(\eta_{+}\right) \epsilon_{A}(\eta)}\right) \frac{\tanh \left(\eta_{+} / 2 T\right)-\tanh (\eta / 2 T)}{\epsilon_{R}\left(\eta_{+}\right)-\epsilon_{A}(\eta)+i / \tau}\right. \\
+ & \tanh \left(\frac{\eta}{2 T}\right)\left[\left(1-\frac{\eta \eta_{+}+\Delta^{2}}{\epsilon_{R}(\eta) \epsilon_{R}\left(\eta_{+}\right)}\right)\left(\epsilon_{R}(\eta)+\epsilon_{R}\left(\eta_{+}\right)+i / \tau\right)^{-1}\right. \\
& \left.\left.+\left(1-\frac{\eta \eta_{-}+\Delta^{2}}{\epsilon_{A}(\eta) \epsilon_{A}\left(\eta_{-}\right)}\right)\left(\epsilon_{A}(\eta)+\epsilon_{A}\left(\eta_{-}\right)-i / \tau\right)^{-1}\right]\right\}
\end{aligned}
$$

The real and imaginary parts of $K^{R}(\omega)$ for $\omega<2 \Delta$ are given by eqs. (28,29) of the text. See Appendix B for the expressions of the same values and their analysis in the range $\omega>2 \Delta$.

\section{Appendix B Above the Threshold.}

Here we present formal expressions for the real and imaginary parts of the normalized Pippard kernel in the range $\omega>2 \Delta$ and a brief analysis of their behavior at zero temperature. The required expressions can be extracted straightforwardly from the general equation (A.13):

$$
\begin{array}{r}
\mathcal{R e K}(\omega)=\int_{-\Delta}^{\Delta} \frac{d \eta}{\epsilon_{+}^{2}+(\bar{\varepsilon}+1 / \tau)^{2}}\left[\epsilon_{+}+\left(\bar{\varepsilon}+\frac{1}{\tau}\right) \frac{\eta_{+} \eta+\Delta^{2}}{\epsilon_{+} \bar{\varepsilon}}\right] \tanh \frac{\eta_{+}}{2 T}+ \\
\int_{\Delta}^{\infty}\left[\frac{\epsilon_{+}+\epsilon}{\left(\epsilon_{+}+\epsilon\right)^{2}+1 / \tau^{2}}\left(1-\frac{\eta_{+} \eta+\Delta^{2}}{\epsilon_{+} \epsilon}\right)\left(\tanh \frac{\eta_{+}}{2 T}+\tanh \frac{\eta}{2 T}\right)+\right. \\
\left.\frac{\epsilon_{+}-\epsilon}{\left(\epsilon_{+}-\epsilon\right)^{2}+1 / \tau^{2}}\left(1+\frac{\eta_{+} \eta+\Delta^{2}}{\epsilon_{+} \epsilon}\right)\left(\tanh \frac{\eta_{+}}{2 T}-\tanh \frac{\eta}{2 T}\right)\right] \frac{d \eta}{2}+
\end{array}
$$




$$
\begin{aligned}
& \int_{\Delta}^{\omega-\Delta} \frac{d \eta}{2}\left[\frac{\epsilon-\epsilon_{-}}{\left(\epsilon-\epsilon_{-}\right)^{2}+1 / \tau^{2}}\left(1+\frac{\eta-\eta+\Delta^{2}}{\epsilon_{+} \epsilon}\right) \tanh \frac{\eta}{2 T}+\right. \\
& \left.\frac{1}{2} \frac{\epsilon+\epsilon_{-}}{\left(\epsilon+\epsilon_{-}\right)^{2}+1 / \tau^{2}}\left(1-\frac{\eta \eta_{-}+\Delta^{2}}{\epsilon \epsilon_{-}}\right)\left(\tanh \frac{\eta}{2 T}-\tanh \frac{\eta-}{2 T}\right)\right] \\
& \mathcal{I} m K(\omega)=\frac{1}{2 \tau} \int_{\Delta}^{\omega-\Delta} d \eta\left[\frac{\tanh (\eta / 2 T)}{\left(\epsilon-\epsilon_{-}\right)^{2}+1 / \tau^{2}}\left(1+\frac{\eta_{-} \eta+\Delta^{2}}{\epsilon_{-} \epsilon}\right) \quad-\right. \\
& \left.\frac{1}{2} \frac{1}{\left(\epsilon+\epsilon_{-}\right)^{2}+1 / \tau^{2}}\left(1-\frac{\eta \eta_{-}+\Delta^{2}}{\epsilon \epsilon_{-}}\right)\left(\tanh \frac{\eta}{2 T}-\tanh \frac{\eta_{-}}{2 T}\right)\right]+ \\
& \frac{1}{2 \tau} \int_{\Delta}^{\infty} d \eta\left[\left(1-\frac{\eta_{+} \eta+\Delta^{2}}{\epsilon_{+} \epsilon}\right)\left(\left(\epsilon_{+}+\epsilon\right)^{2}+\frac{1}{\tau^{2}}\right)^{-1}-\right. \\
& \left.\left(1+\frac{\eta_{+} \eta+\Delta^{2}}{\epsilon_{+} \epsilon}\right)\left(\left(\epsilon_{+}-\epsilon\right)^{2}+\frac{1}{\tau^{2}}\right)^{-1}\right]\left(\tanh \frac{\eta_{+}}{2 T}-\tanh \frac{\eta}{2 T}\right)
\end{aligned}
$$

The notations are the same as in the text.

Let us first take a look at the normal limit $\Delta \longrightarrow 0$. In this case the first integral in the 1.h.s. of the eq. (B.1) just vanishes whereas the coherence factors $\left(1 \pm\left(\eta \eta_{ \pm}+\Delta^{2}\right) / \epsilon \epsilon_{ \pm}\right)$are reduced either to 0 or to 2 due to the following identities: $\epsilon_{+}=\eta_{+}, \epsilon=\eta$ and $\epsilon_{-}=-\eta_{-}$. The Lorentzian factors associated with the non-vanishing coherence factors tend to $\omega /\left(\omega^{2}+1 / \tau^{2}\right)$ or $(1 / \tau) /\left(\omega^{2}+1 / \tau^{2}\right)$ in formulas for the real and the imaginary parts of the kernel respectively. Taking into account the above remarks one can evaluate the remaining integrals straightforwardly and finally arrive to the DrudeLorentz formula (31).

We specially consider the case of zero temperature. Then equations (B.1) and (․2) look much simpler:

$$
\begin{array}{r}
\mathcal{R} e K(\omega)=\int_{\Delta}^{\infty} \frac{\epsilon_{+}+\epsilon}{\left(\epsilon_{+}+\epsilon\right)^{2}+1 / \tau^{2}}\left(1-\frac{\eta_{+} \eta+\Delta^{2}}{\epsilon_{+} \epsilon}\right) d \eta+ \\
\frac{1}{2} \int_{\Delta}^{\omega-\Delta} d \eta\left[\frac{\epsilon-\epsilon_{-}}{\left(\epsilon-\epsilon_{-}\right)^{2}+1 / \tau^{2}}\left(1+\frac{\eta_{-} \eta+\Delta^{2}}{\epsilon_{-} \epsilon}\right)+\right. \\
\left.\frac{\epsilon+\epsilon_{+}}{\left(\epsilon+\epsilon_{+}\right)^{2}+1 / \tau^{2}}\left(1-\frac{\eta_{+} \eta+\Delta^{2}}{\epsilon_{+} \epsilon}\right)\right]+ \\
\int_{-\Delta}^{\Delta}\left[\epsilon_{+}+\left(\bar{\varepsilon}+\frac{1}{\tau}\right) \frac{\eta_{+} \eta+\Delta^{2}}{\epsilon_{+} \bar{\varepsilon}}\right] \frac{d \eta}{\left(\epsilon_{+}^{2}+(\bar{\varepsilon}+1 / \tau)^{2}\right)}
\end{array}
$$




$$
\begin{aligned}
\mathcal{I} m K(\omega)=\frac{1}{2 \tau} \int_{\Delta}^{\omega-\Delta} & {\left[\left(1+\frac{\eta_{-} \eta+\Delta^{2}}{\epsilon_{-} \epsilon}\right)\left(\left(\epsilon-\epsilon_{-}\right)^{2}+\frac{1}{\tau^{2}}\right)^{-1}-\right.} \\
& \left.\left(1-\frac{\eta_{-} \eta+\Delta^{2}}{\epsilon_{-} \epsilon}\right)\left(\left(\epsilon+\epsilon_{-}\right)^{2}+\frac{1}{\tau^{2}}\right)^{-1}\right] d \eta
\end{aligned}
$$

The imaginary part of (B.4) corresponds to the absorption of a photon accompanied by the creation of two quasiparticles (dissociation of a Cooper pair). The probability of this process, suppressed in the absence of scatterers, is proportional to the scattering rate $1 / \tau$. In addition to the coherence factor it contains the Lorentzian distribution function for the total momentum of a pair of quasiparticles located on the opposite points of a diameter. When $\tau \rightarrow \infty$, this distribution turns into $\delta$-function.

The integral in the above formula (B.4) for the imaginary part may be expressed in terms of the standard elliptic integrals as follows:

$$
\begin{gathered}
\mathcal{I} m K^{R}(w)=\frac{4 \Delta^{2} \tau \Pi(n(\omega, \tau), m(\tau))}{(\omega+2 \Delta)\left(1-4 \Delta^{2} \tau^{2}+\omega^{2} \tau^{2}\right)}\left(1-\frac{\left(2 \Delta \omega \tau^{2}\right)^{2}}{\left(1+\omega^{2} \tau^{2}\right)^{2}}\right) \\
-\frac{(\omega+2 \Delta) \tau}{1+\omega^{2} \tau^{2}} E(m(\omega))+\frac{4 \Delta \tau\left(\Delta+\omega+\omega^{3} \tau^{2}\right)}{(\omega+2 \Delta)\left(1+\omega^{2} \tau^{2}\right)^{2}} K(m(\omega))
\end{gathered}
$$

Here $\mathrm{K}(m), \mathrm{E}(m)$ and $\Pi(n, m)$ are complete elliptic integrals of the first, the second and the third kind respectively. The moduli of the integrals depend on the frequency and the scattering rate.

$$
m(\omega)=\left(\frac{\omega-2 \Delta}{\omega+2 \Delta}\right)^{2}, \quad n(\omega, \tau)=-\frac{\tau^{2}(\omega-2 \Delta)^{2}\left(1+\omega^{2} \tau^{2}\right)}{\left(1+\left(\omega^{2}-4 \Delta^{2}\right) \tau^{2}\right)}
$$

The function (B.5) is always negative. Just above the threshold its absolute value increases linearly with the intercept being inversely proportional to the scattering rate:

$$
\mathcal{I} m K(\omega)=-\pi \tau(\omega-2 \Delta) / 2+\mathrm{O}(\omega / \Delta-2)^{2}
$$

The non-linear phase of the descending slope may be approximated by means of the following interpolating function:

$$
\mathcal{I} m K(\omega) \approx \frac{\pi}{4 \Delta \tau}\left(\frac{1}{\sqrt{1+4(\omega-2 \Delta) \Delta \tau^{2}}}-1\right)
$$


This interpolation gives a remarkably good estimate of the actual $\mathcal{I} m K(\omega)$ almost up to the point $\omega_{\min } \approx 2 \Delta+0.9 / \tau$ where the imaginary part reaches its minimum [1. A minimal value of $\operatorname{Im} K(\omega)$ significantly depends on the scattering rate. If the latter is small compared to the threshold frequency $(\Delta \tau \gg 1)$ the dissipation is also small: $\operatorname{Im} K\left(\omega_{\min }\right) \approx-\pi / 4 \Delta \tau$. However, in the regime of a strong scattering the dissipation growth is saturated at the level:

$$
\lim _{\Delta \tau \rightarrow 0} \mathcal{I} m K\left(\omega_{\text {min }}\right)=-\frac{1}{2}
$$

Examining eq. (B.8) one can easily see the appearance of the intermediate frequency scale scale $1 / \Delta \tau^{2}$.

In the high frequency range the imaginary part of the Pippard kernel decays monotonically with the asymptotics:

$$
\operatorname{Im} K(\omega)=-1 / \omega \tau+\mathrm{O}\left(1 / \omega^{2}\right)
$$

in conformity with the Drude-Lorentz formula for the normal metal (31). The existence of the gap becomes irrelevant for the plasma oscillations driven by the electromagnetic wave provided the frequency of the latter is large compared to the threshold value.

The discontinuity of the derivative with respect to frequency of the imaginary part of the Pippard kernel at $\omega=2 \Delta$ implies that the total Pippard kernel, considered as a function of the complex variable $\omega$, has a logarithmictype singularity at this point. The behavior of this function in the vicinity of its singularity is governed by the eq. (35). The real part of the kernel decreases rapidly when approaching the threshold with a negative logarithmically divergent derivative.

Switching the temperature on causes the augmentation of the dissipation. On the contrary, $\operatorname{Re} K(\omega)$ at finite temperature is diminished. Still near the threshold the asymptotic behavior $(\mathbb{B . 8})$ is valid if one considers this contribution as complementary to the Cherenkov absorption part, and multiplies it by the factor $\tanh (\Delta / 2 T)$.

\section{References}

\footnotetext{
${ }^{7}$ The approximate dependence of the minimum position on a scattering rate has been found numerically.
} 
[1] B. Koch, M.Durler, H.P. Geserich, Th.Wolf, G.Roth and G.Zachman, in Electronic Properties of High- $T_{c}$ Superconducting and Related Compounds. H. Kuzmany, M. Mehring and J.Fink (Eds.) (1990).

[2] D. de Marel et al, Physica 176C (1991) 1.

[3] K. Tamasaku, Y. Nakamura, and S. Uchida, Phys. Rev. Lett. 69 (1992) $1455-1458$.

[4] T. Mishonov, Dubna preprint, 1992; Phys. Rev. B, in press.

[5] M. Tachiki, T. Koyama, and S. Takahashi, Preprint of the Institute for Material Research, Tohoku University, (1994).

[6] V. L. Pokrovsky and S. K. Savvinykh, Sov. Phys. JETP16 (1963), 404.

[7] G. M. Eliashberg, Sov.Phys. JETP 34 (1972), 668-676.

[8] A. A. Abrikosov, L. P. Gorkov, I. E. Dzyaloshinskii, Methods of Quantum Field Theory in Statistical Mechanics, Addison-Wesley, NY,1965.

[9] A. A. Varlamov and D. V. Livanov, Sov.Phys.JETP 71(1990) 325-330.

[10] R. Klemm, A. Luther, and M. R. Beasley, Phys. Rev. B44 (1991) 877890.

[11] L. V. Keldysh, ZhETF, 47 (1964), 1515; Sov. Phys.JETP 20 (1965), 1018 .

[12] D. C. Mattis and J. Bardeen, Phys.Rev.111 (1958), 412-417.

[13] A. A. Abrikosov, L. P. Gor'kov, and I. M. Khalatnikov, ZhETF35 (1958) 265-275; Sov. Phys. JETP 35(8) (1959) 182-189. ZhETF 37 (1959) 319.

[14] G. Rickayzen. Theory of Superconductivity. Interscience Publishers. New York, 1965.

[15] See for example T. Ito et al., Nature 334 (1991), 596; Y. Nakamura and S. Uchida, Phys. Rev. B47 (1993), 8369-72. 


\section{Figure Captions.}

\section{Figure 1}

Reflectivity versus frequency (measured in relative units: $\omega / \Omega_{z}$ ) in the normal state.

Figure 1A: The high-frequency dielectric constant $\mathcal{E}=25$.

Figure 1B: The high-frequency dielectric constant $\mathcal{E}=4$.

The different curves on both plots correspond to the different values of scattering rate $\Omega_{z} \tau=0.01 ; 0.1 ; 0.5 ; 1.0 ; 5.0$.

\section{Figure 2}

Real part of the normalized Pippard kernel versus frequency (measured in relative units: $\left.\omega / T_{c}\right)$. The bare plasma frequency $\Omega_{z}=1.2 T_{c}$. The highfrequency dielectric constant $\mathcal{E}=25$. Three spectra are plotted for each of the following scattering rates:

Figure 2A: $\tau T_{c}=0.1$

Figure 2B: $\tau T_{c}=3.4$

The dashed plot corresponds to $T / T_{c}=0.30, \Delta / T_{c}=1.73$ and the dasheddotted one corresponds to $T / T_{c}=0.74, \Delta / T_{c}=1.38$ respectively. The values of $\Delta\left(T / T_{c}\right)$ have been calculated in the frameworks of the BCS theory.

\section{Figure 3}

Imaginary part of the normalized Pippard kernel. The bare plasma frequency $\Omega_{z}=1.2 T_{c}$. The high-frequency dielectric constant $\mathcal{E}=25$, The dashed plot corresponds to $T / T_{c}=0.30, \Delta / T_{c}=1.73$ and the dashed-dotted one corresponds to $T / T_{c}=0.74, \Delta / T_{c}=1.38$.

Figure 3A: $\tau T_{c}=0.1$.

Figure 3B: $\tau T_{c}=3.4$ 


\section{Figure 4}

Reflectivity versus frequency (measured in relative units: $\omega / T_{c}$ ). The bare plasma frequency $\Omega_{z}=1.2 T_{c}$. High-frequency dielectric constant $\mathcal{E}=25$. Three spectra are plotted for each of the following scattering rates:

Figure 4A: $\tau T_{c}=0.1$.

Figure 4B: $\tau T_{c}=0.9$

Figure $4 \mathrm{C}: \tau T_{c}=1.7$

Figure 4D: $\tau T_{c}=3.4$

The solid curve is a plot for the normal state. The dashed plot corresponds to $T / T_{c}=0.30, \Delta / T_{c}=1.73$ and the dashed-dotted one corresponds to $T / T_{c}=0.74, \Delta / T_{c}=1.38$ respectively.

Figure 5 Comparison of the imaginary parts of the normalized Pippard kernel for the BCS and the two-fluid models. The solid curves correspond to the BCS model. The dashed plots correspond to the two-fluid model. The bare plasma frequency $\Omega_{z}=1.2 T_{c}$. High-frequency dielectric constant $\mathcal{E}=25, T / T_{c}=0.30, \Delta / T_{c}=1.73$.

Figure 5A: $\tau T_{c}=0.1$.

Figure 5B: $\tau T_{c}=3.4$ 

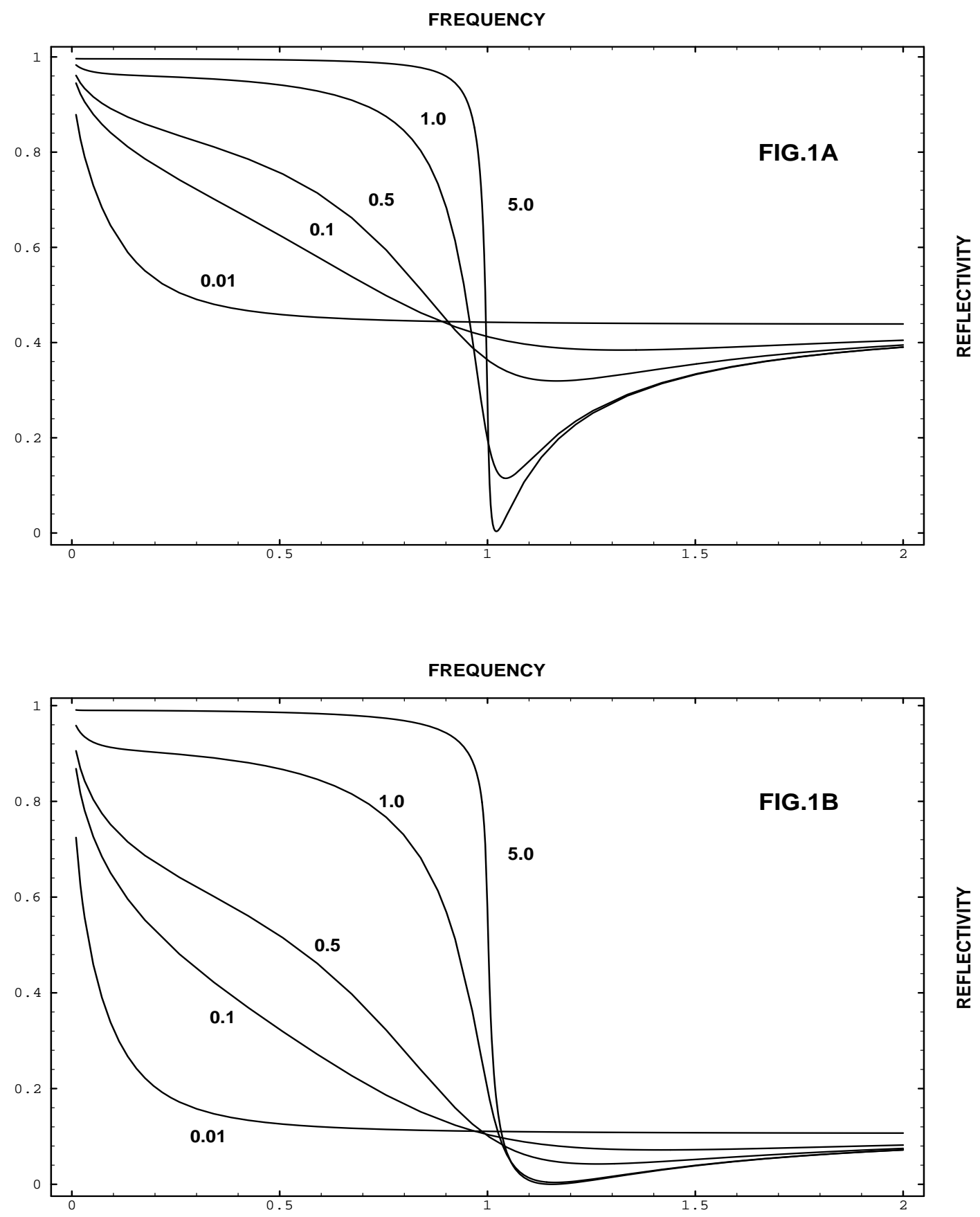

站 

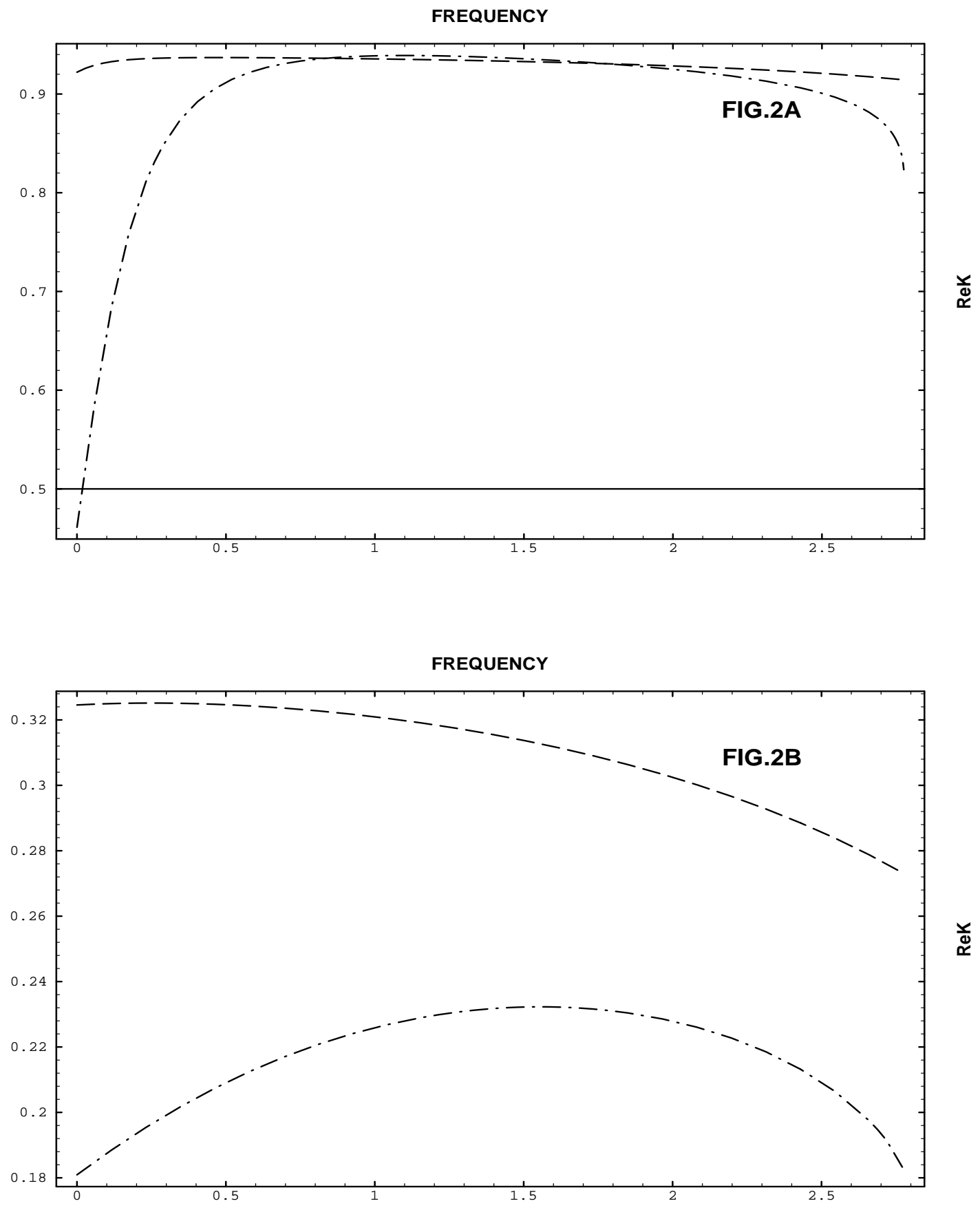


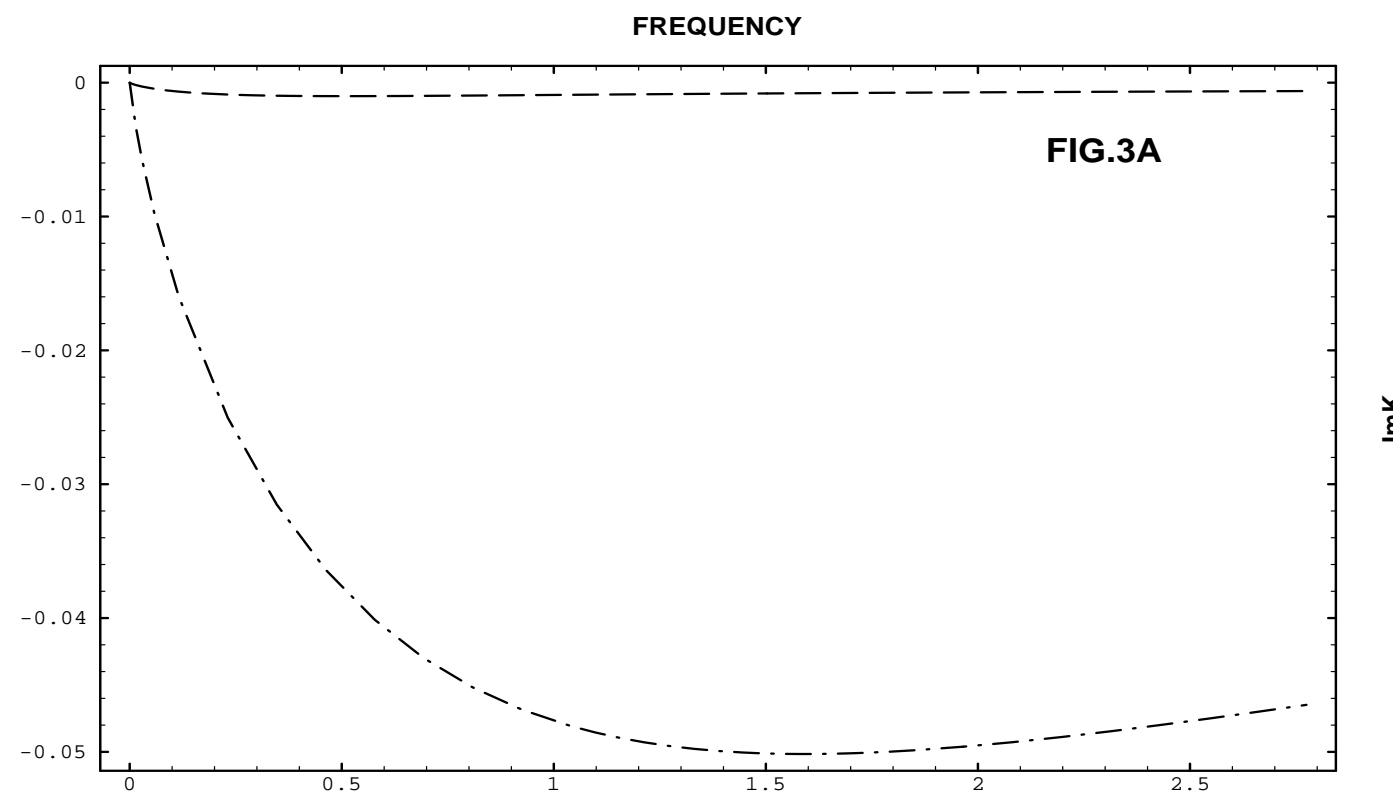

FREQUENCY

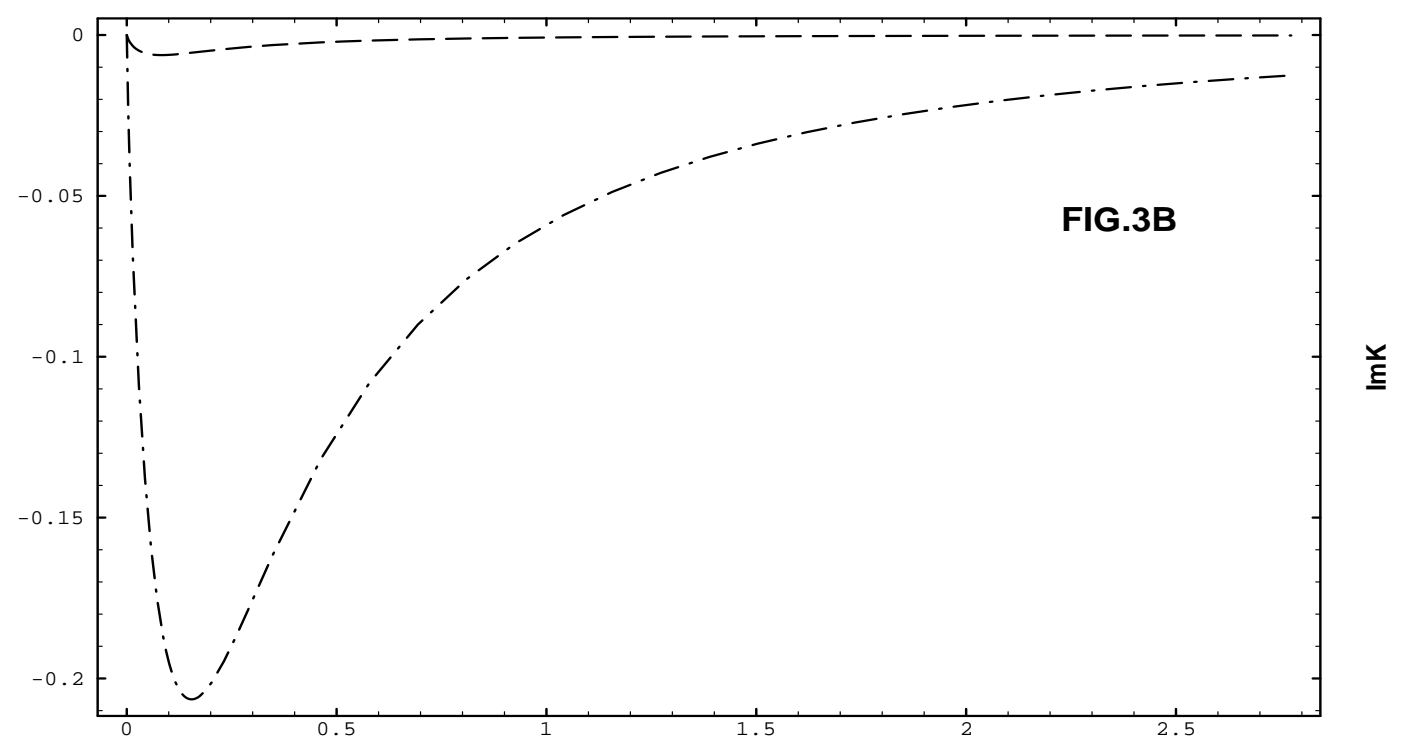


FREQUENCY

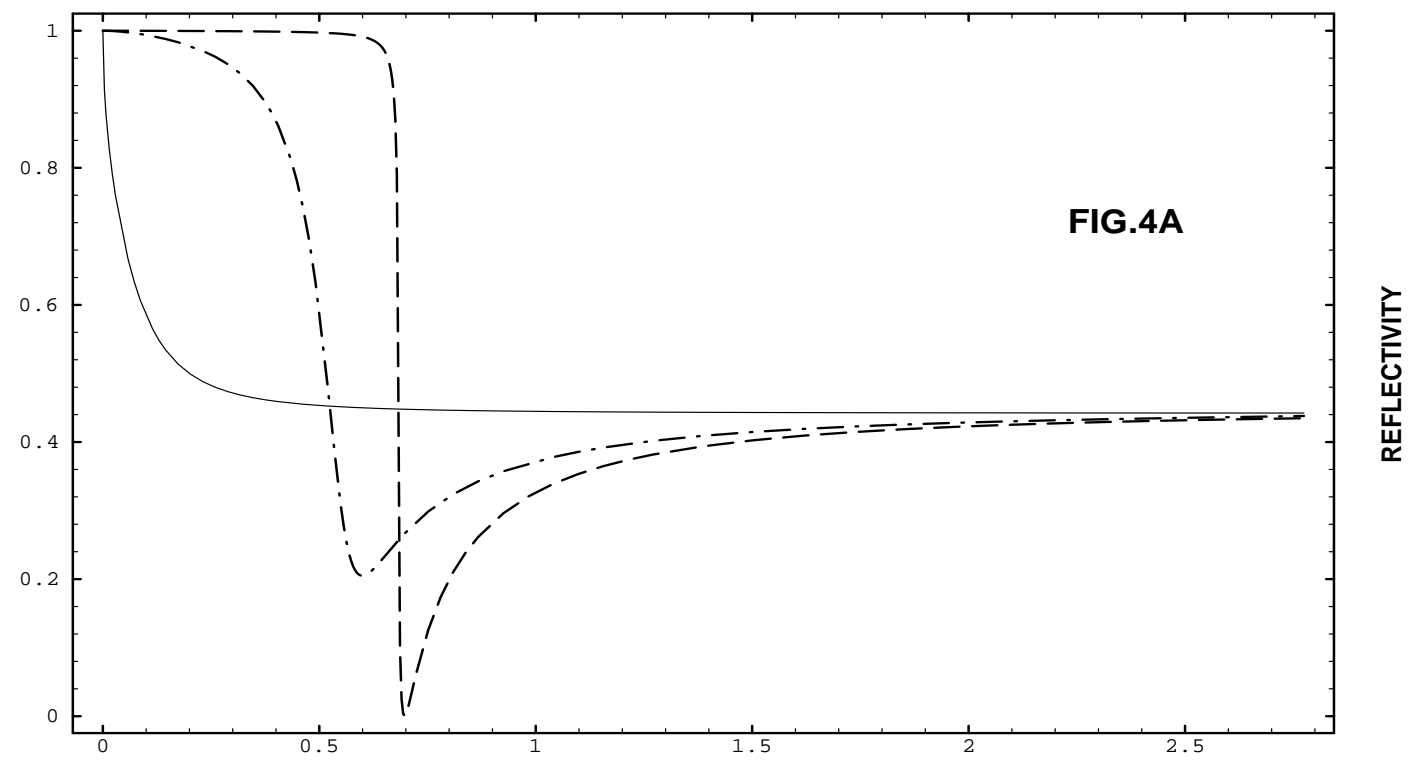

FREQUENCY

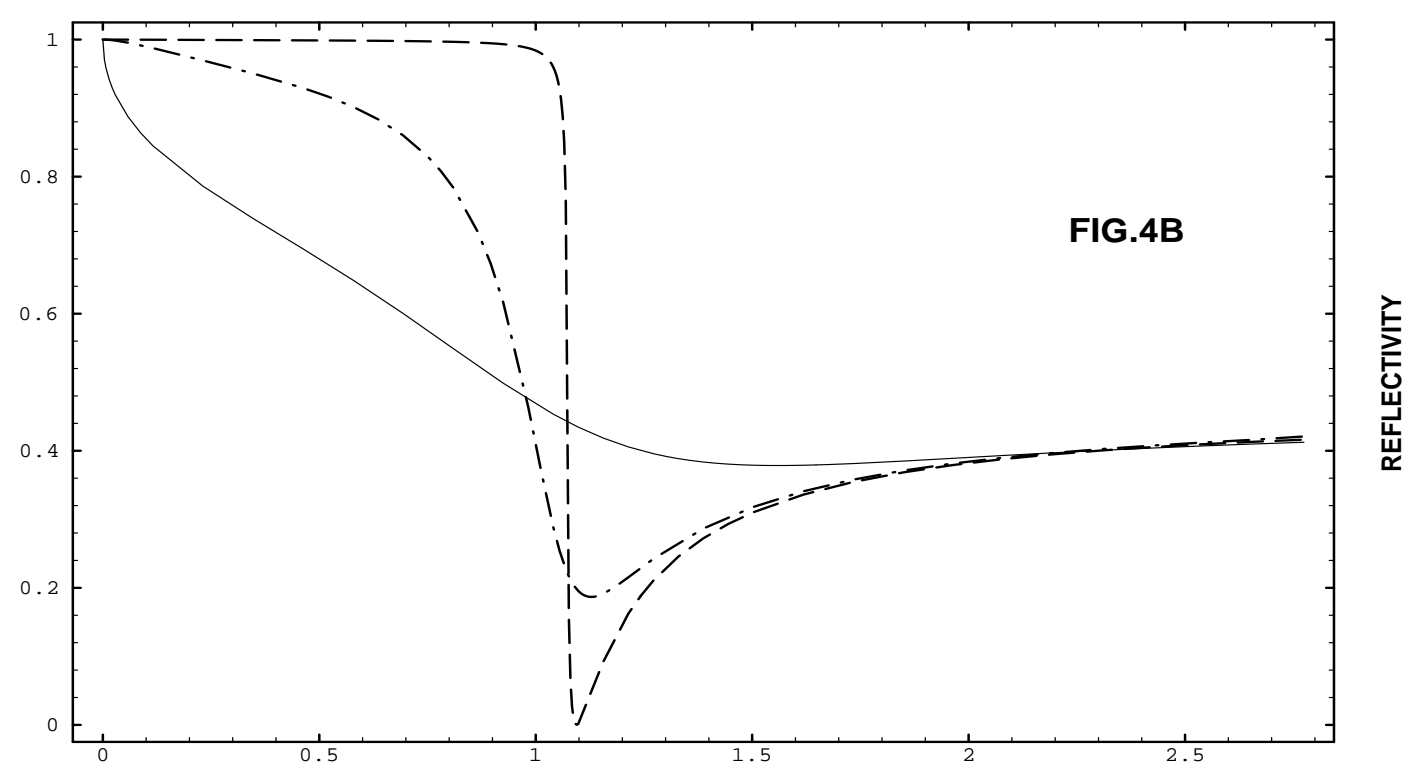



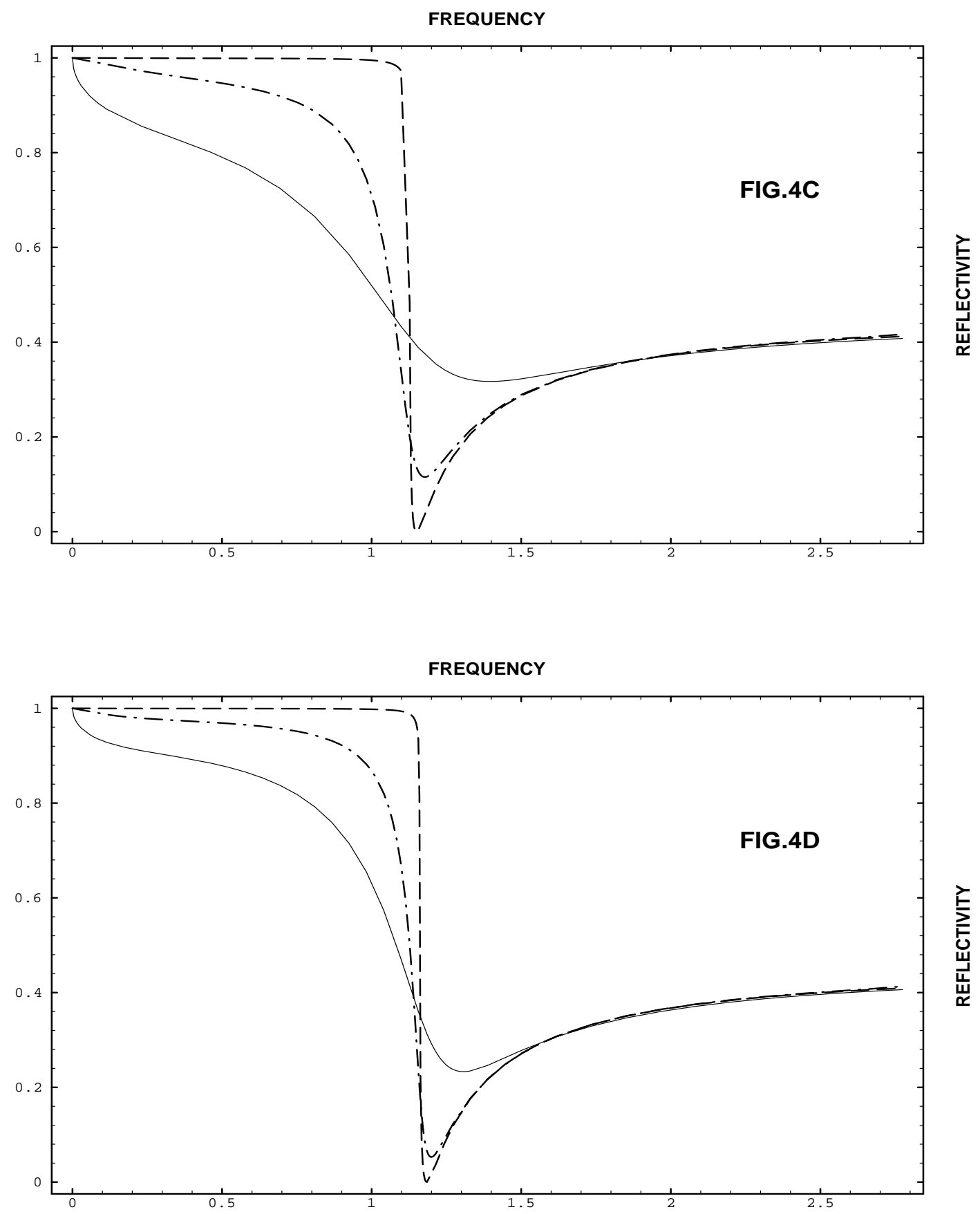

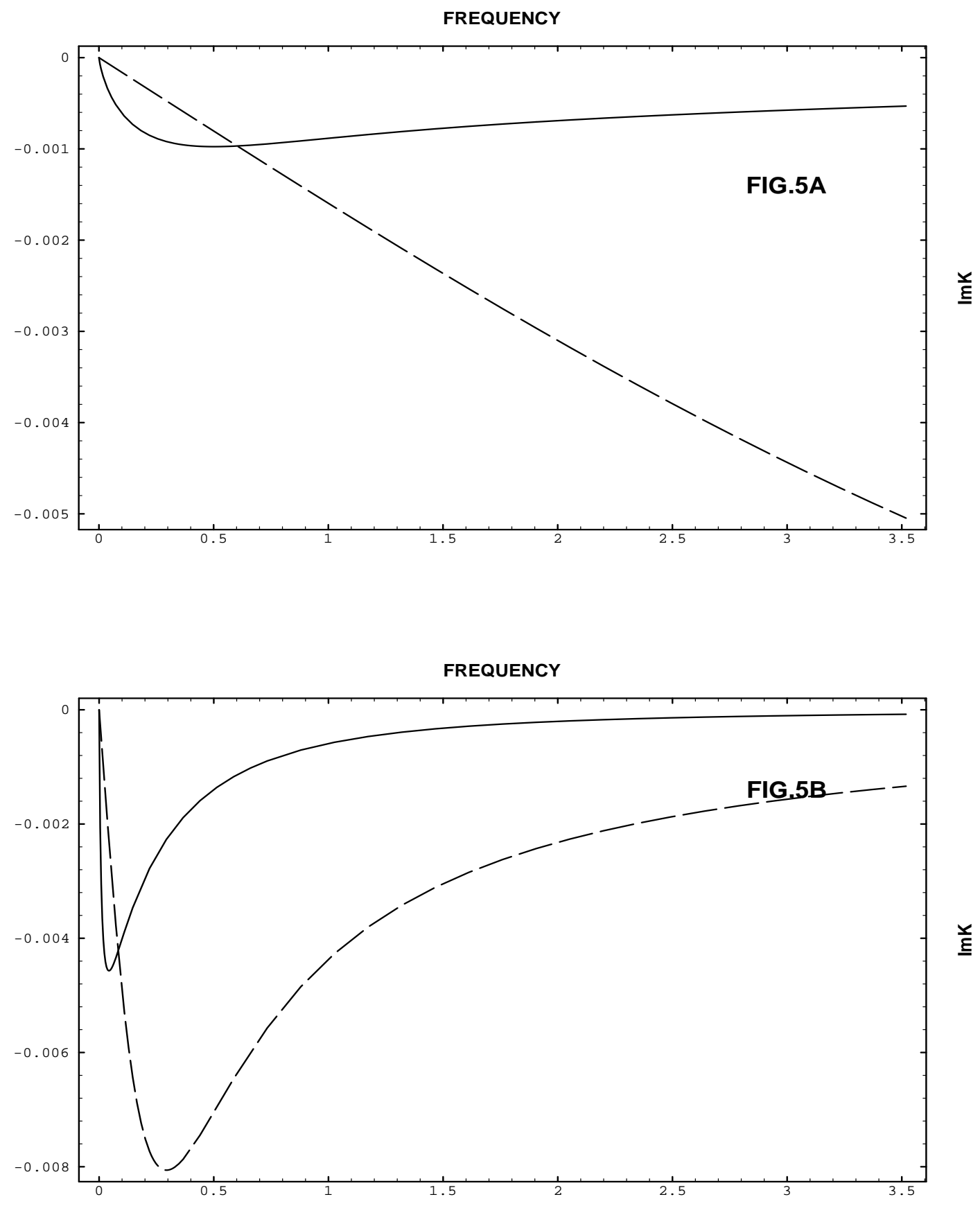
FREQUENCY

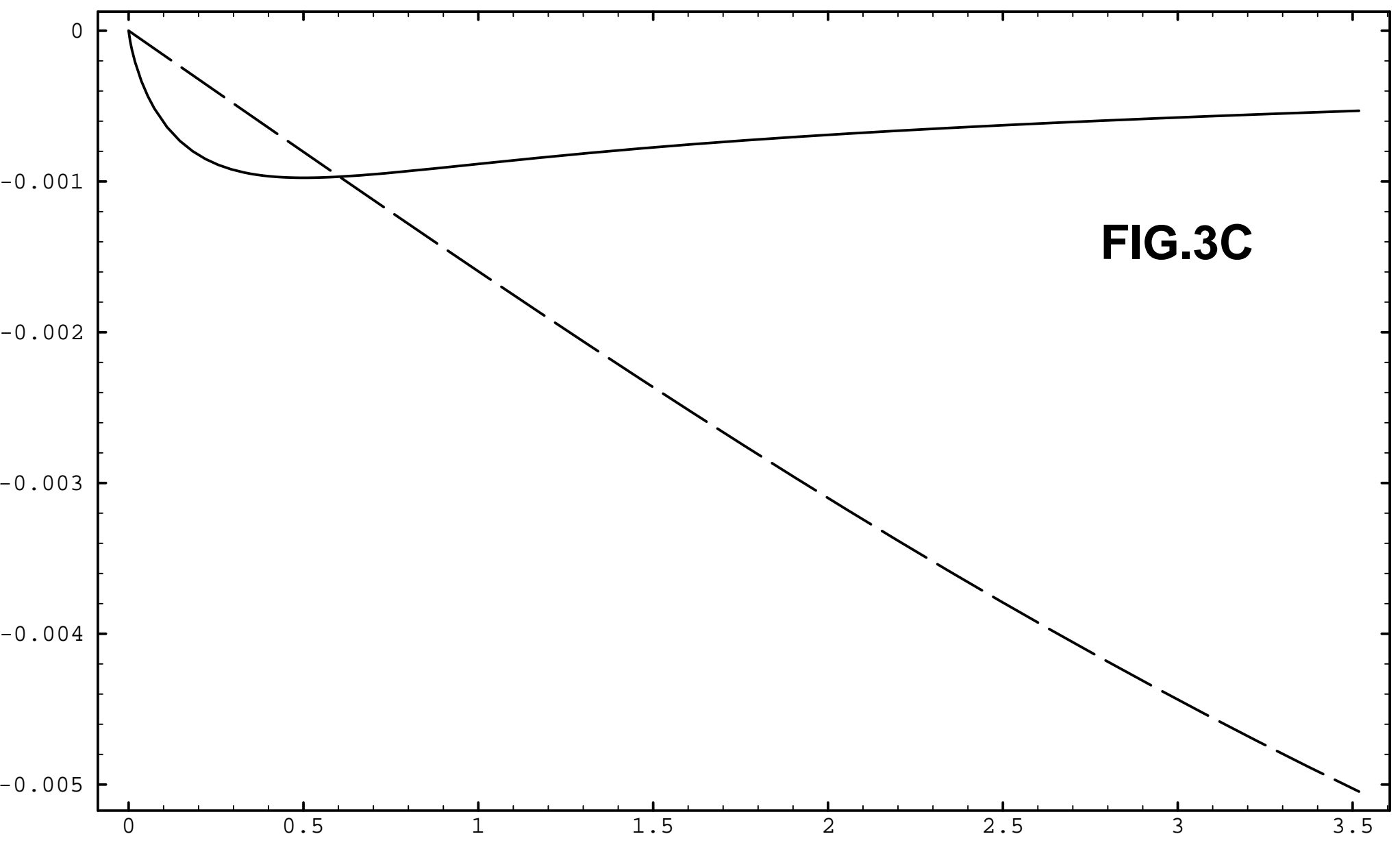

